\title{
Acidic Leucine-Rich Nuclear Phosphoprotein 32 Family Member B
}

National Cancer Institute

\section{Source}

National Cancer Institute. Acidic Leucine-Rich Nuclear Phosphoprotein 32 Family Member

B. NCI Thesaurus. Code C25962.

Acidic leucine-rich nuclear phosphoprotein 32 family member B (251 aa, $29 \mathrm{kDa}$ ) is encoded by the human ANP32B gene. This protein is involved in the regulation of both the cell cycle and apoptosis. 